\title{
Should Propofol and Alfentanil Be Combined in Patient-Controlled Sedation? A Randomised Controlled Trial Using Pharmacokinetic Simulation
}

\author{
Andreas Nilsson1,2, Lena Nilsson ${ }^{1,2}$, Thomas Schnider ${ }^{3}$, Eva Uustal ${ }^{4}$, Folke Sjöberg5 \\ ${ }^{1}$ Department of Medical and Health Sciences, Linköping University, Linköping, Sweden \\ ${ }^{2}$ Department of Anesthesiology and Intensive Care, County Council of Östergötland, Linköping, Sweden \\ ${ }^{3}$ Institute of Anaesthesiology, Kantonsspital St. Gallen, St. Gallen, Switzerland \\ ${ }^{4}$ Departmentof Obstetrics and Gynecology, Linköping University, Linköping, Sweden \\ ${ }^{5}$ Department of Clinical and Experimental Medicine, Linköping University, Linköping, Sweden \\ Email: andreas.nilsson@regionostergotland.se
}

Received 29 April 2015; accepted 21 June 2015; published 24 June 2015

Copyright (C) 2015 by authors and Scientific Research Publishing Inc.

This work is licensed under the Creative Commons Attribution International License (CC BY). http://creativecommons.org/licenses/by/4.0/

(c) (i) Open Access

\section{Abstract}

Background: Patient-controlled sedation (PCS) is increasingly used for moderate sedation. Detailed understanding is essential for maintaining safety and giving the most benefit. We wanted to explore the associations between patients' characteristics, perioperative pain and anxiety, the procedure, and the calculated concentrations at the effect site $\left(\mathrm{C}_{\mathrm{e}}\right)$ of propofol. We also wanted to analyse the pharmacokinetic profiles of propofol and alfentanil during PCS, and their association with respiratory complications. Methods: 155 patients were double-blinded and randomised to have propofol or propofol and alfentanil for PCS during gynaecological surgery. Pharmacokinetic simulation of $\mathrm{C}_{e}$ and multiple regressions aided the search for correlations between explanatory variables and concentrations of drugs. Results: In group propofol, treatment for incontinence, anterior repair, and the patient's weight correlated the best $\left(\mathrm{B}-\mathrm{coef}=0.20,0.20\right.$ and $0.01 ; \mathrm{r}=0.69 ; \mathrm{r}^{2}$ $=0.48$ ). When alfentanil was added, alfentanil and the patient's weight were associated with $C_{e}$ of propofol ( $B$-coef $=-0.40$ and $0.01 ; r=0.70 ; r^{2}=0.43$ ). Logistic regression indicated that age and $C_{e}$ of drugs were related to ten cases of respiratory complications. Conclusions: Patients' weights and the type of surgery performed were associated with the $C_{e}$ of propofol; this knowledge could be used for refinement of the doses given during PCS. Because the pharmacokinetic profiles of propofol and alfentanil are different, the alfentanil effect becomes predominant during the time course of sedation. In order to reduce the risk of early and late respiratory depression, alfentanil should not be added to propofol in the same syringe. 


\section{Keywords}

\section{Anaesthetics, Intravenous, Propofol, Analgesics, Opioids, Alfentanil, Sedation}

\section{Introduction}

Minor procedures can be managed successfully using local anaesthesia and moderate sedation with propofol [1], and American [2] and European guidelines [3] give specific recommendations for the use of propofol by nonanaesthetists. Although adverse events are rare, sedation with propofol includes the risk of cardiorespiratory instability [4]. Propofol given for patient-controlled sedation (PCS) is an option for moderate sedation, and is often combined with opioids for the control of pain [5]-[7], although respiratory events have been reported when remifentanil [8] or alfentanil [9] were added to propofol. Propofol and opioids mixed in the same syringe for PCS during an operation has been connected with respiratory events [10]. Knowledge about accumulated doses of propofol and opioids, and the factors that explain why patients request repeated doses, is limited, but could help to improve safety. To refine the system of PCS so that it will give a better clinical performance, we need further information about the pharmacokinetic profiles of the drugs used, as well as what factors influence the size of each dose and the need for repeated doses.

In this paper we have recalculated data from a recent publication [10] in which we conclude that a mixture of alfentanil and propofol for PCS increases the risk of respiratory problems and therefore requires the presence of an anaesthetist in the operating room. Our main objectives were to analyse the variables that could explain the concentrations of propofol at the effect site $\left(\mathrm{C}_{\mathrm{e}}\right)$ and the pharmacokinetic profile derived from the combined use of propofol and alfentanil. We first hypothesised that the physical characteristics of the patients, the type of operation, and the degrees of perioperative pain and anxiety could be used to explain the calculated $\mathrm{C}_{\mathrm{e}}$ of drugs used. Secondly, we hypothesised that the pharmacokinetic profiles from the simulation of concentrations of propofol and alfentanil could explain the respiratory depression that occurred in a subgroup of patients [10].

\section{Methods}

Details of the design, methods, inclusion and exclusion criteria, patients, and results of the original study have been described previously [10]. After earning approval from the local ethics committee and written consent from all the patients, the study included 165 gynaecological patients who were treated in an outpatient clinic for incontinence, anterior or posterior repair of the vaginal wall, or combinations of reconstructive genital surgery. In a prospective double-blind trial, the patients were randomised to use propofol, or propofol with alfentanil, as PCS. A nurse anaesthetist was present and responsible for the protocol, operative monitoring, the PCS pump, and specific interventions to ensure the safety of the patients. A consultant anaesthetist was available within a few minutes. 155 patients used PCS for the entire procedure as ten patients expressed their need for assistance from the nurse anaesthetist and were given additional doses of propofol.

Local anaesthesia was used for pain control during the operation and was given after the patient had made several demands on the dose button and felt sedated. The infiltration of the local anaesthetic is unpleasant and painful, and the physical relaxation from the moderate sedation facilitates infiltration. The operations could also involve moments of pain or discomfort in areas where the infiltration was only partially effective. After induction, patients were instructed to use the PCS pump to keep themselves comfortable or to reduce the effects of short moments of pain. Patients with more than two episodes of pain were treated with complementary infiltrations. Postoperatively, the patients were supervised in the recovery room for at least 90 minutes before they were discharged.

Cardiorespiratory vital signs were monitored before sedation began, and data were recorded continuously or at 5-minute intervals during the procedure. Perioperative pain and anxiety were recorded using visual analogue scales (VAS). Patients marked their maximum experience on a $100 \mathrm{~mm}$ line on which 0 indicated no pain or anxiety and 100 indicated unbearable pain or anxiety. Peroperative pain was rated at the beginning and the end of the procedure when the patients were awake. If the patients felt pain during the operation, then it was recorded, and if they were able to communicate, the intensity was measured as well. Anxiety was rated preoperatively and postoperatively within an hour of completion of the operation. 
The PCS pump (IVAC 5000 PCAM-pump, Cardinal Health, Dublin, UK) was programmed to deliver either propofol $4.25 \mathrm{mg}$ or a mixture of propofol $4.25 \mathrm{mg}$ and alfentanil $0.0375 \mathrm{mg}$ each time the patient presses the delivery button. There was no lock-out period, but the capacity of the pump restricted the maximum number of doses to five per minute. The addition of alfentanil was made via double-blind randomisation. The code was broken at the end of the study.

The pharmacokinetic simulation was based on the dosing history recorded with the PCS pump (the time that the fixed bolus doses were given). For propofol, we used the pharmacokinetic models described by Schnider et al. [11] [12], which use age, height, weight, and sex as covariates. The pharmacokinetic profiles of alfentanil were simulated from the model described by Scott et al. [13], which was scaled by weight. For both drugs the $\mathrm{C}_{\mathrm{e}}$ were calculated with the software programme Excel (Microsoft Corporation) using an Add-In (PKPD Tools for Excel; http://www.pkpdtools.com/doku.php/excel:start).

\section{Statistical Analysis}

For the statistical analysis we used Statistica ${ }^{\circledR}$ version 10 (Stat Soft Inc., Tulsa, USA).

Differences in patients' evaluation of anxiety and pain between the propofol and propofol-and-alfentanil groups were assessed using the non-parametric Mann-Whitney $U$ test. Differences in age and weight were assessed using Student's t-test. We used a multiple regression model to help evaluate how patients' characteristics and data about the procedures were associated with the $\mathrm{C}_{\mathrm{e}}$. We built one model each for propofol and propofol/ alfentanil.

After all the variables had been included, we used stepwise backward multiple regressions. The least significant predictor was excluded, and the overall significance in the model and for each predictive variable was checked again. This was repeated until all the predictive variables achieved significance. We used analysis of variance (ANOVA) to compare the $\mathrm{C}_{\mathrm{e}}$ of propofol and alfentanil among patients with respiratory impairments, those who made the most demands for doses from the PCS pump, and those with the highest calculated $\mathrm{C}_{\mathrm{e}}$, respectively, with the rest of the patients in the groups. Values of $\mathrm{C}_{\mathrm{e}}$ from the first 20 minutes of sedation were used for this comparison. A logistic regression was used to explore the variables that could explain the obstructed airway or apnoea. Weight, age, dose ratio (doses demanded divided by doses delivered), patients' anxiety and pain, and $\mathrm{C}_{\mathrm{e}}$ values of propofol and alfentanil were chosen as predictors. Different models were built, and the model that was considered clinically relevant and the most significant was kept for explanation. Probabilities of less than 0.05 were accepted as significant.

\section{Results}

Patients' age, weight and evaluations of anxiety and pain using visual analogue scales did not differ between the groups (Table 1). The variables that were included in the multiple regression analysis are shown in Table 2. Anterior repair, treatment of incontinence, and the patient's weight were associated with the concentration of propofol $\left(r^{2}=0.48\right)$, which, when alfentanil was added, was not related to any of the procedures. The addition of alfentanil was associated with a decreased concentration of propofol at the site, and heavier body weight influenced the increased concentration of propofol $\left(r^{2}=0.43\right)$. Heavier body weight was also associated with a significantly greater demand for doses during induction $(\mathrm{p}<0.01)$. Self-rated preoperative or peroperative anxiety or pain did not affect the concentrations of drugs used in PCS.

The pharmacokinetic profile of propofol is shown in Figure 1. For all 145 patients there was a short peak in the estimated $\mathrm{C}_{\mathrm{e}}$ of propofol after five minutes, and the concentration was then reduced. Alfentanil had a different profile (Figure 2), in that a plateau was reached after 15 minutes and the concentration was almost constant for another 15 minutes.

Respiration was impaired only in the group given a mixture of alfentanil and propofol. Ten of the 79 patients in that group developed apnoea or obstruction of the airway within the first 10 minutes. The only significant difference in characteristics between the group with respiratory impairments and the rest of the patients given alfentanil was lower weight (62 (11) kg compared with $72(14) \mathrm{kg}, \mathrm{p}=0.04)$. ANOVA from the pharmacokinetic calculation of the $C_{e}$ of propofol showed no significant differences between the patients with respiratory impairment and the remaining patients in the propofol/alfentanil group $(p=0.49)$; there were also no significant differences in the $\mathrm{C}_{\mathrm{e}}$ of alfentanil $(\mathrm{p}=0.40)$. 


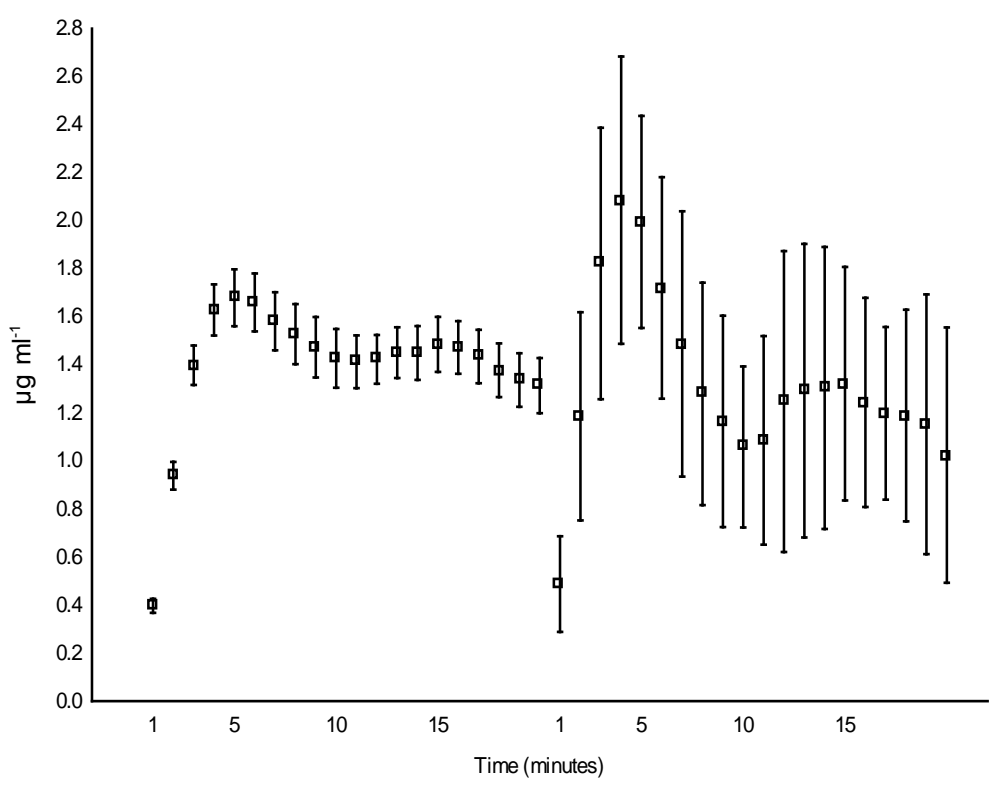

Figure 1. Estimated concentrations of propofol at effect site during the first 20 minutes; comparison between all spontaneously-breathing patients given propofol $(n=145)$ and those with apnoea or obstructed airways $(n=10)$. Mean concentrations of propofol are displayed as boxes, and the bars indicate the $95 \%$ confidence interval (CI). The data in both graphs are from the first 20 minutes of sedation; the first half of the graph indicates the normallybreathing patients and the second half the patients with impaired respiration. Concentrations of propofol decline further after these 20 minutes.

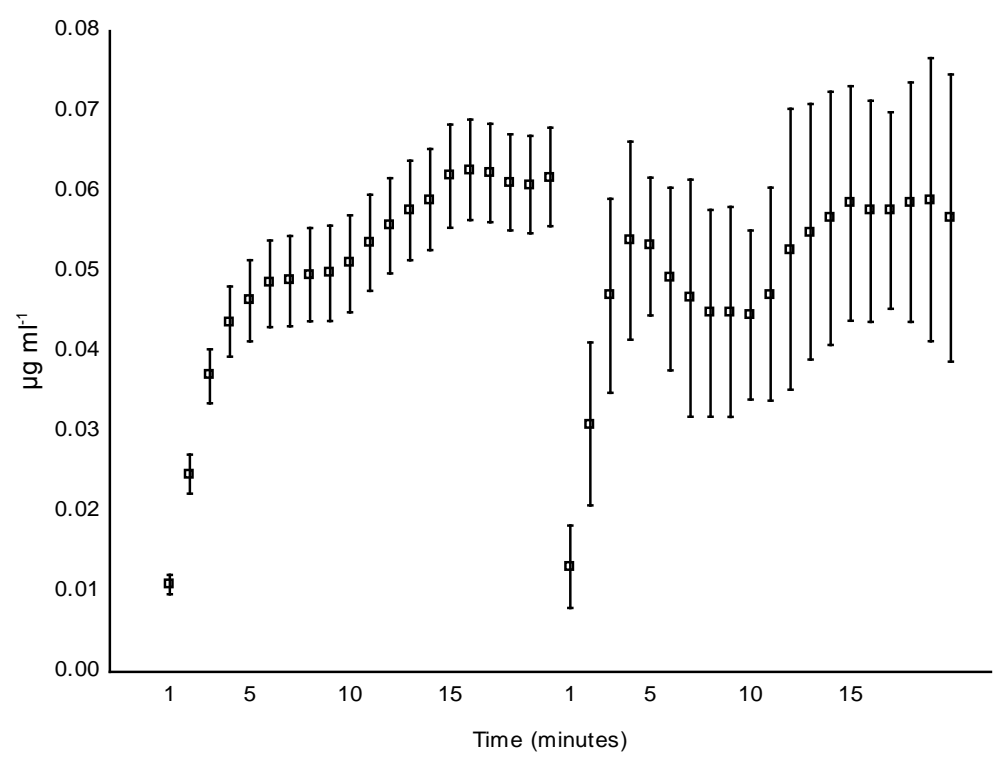

Figure 2. Estimated mean concentrations of alfentanil at effect site during the first 20 minutes; comparison between all spontaneously-breathing patients given a mixture of alfentanil and propofol $(n=69)$ and those with apnoea or obstructed airways $(n=10)$. Mean concentrations of alfentanil are displayed with boxes, and the bars indicate the 95\% confidence interval (CI). The data in both graphs are from the first 20 minutes of sedation; the first half of the graph indicates the normally-breathing patients and the second half the patients with impaired respiration. The 20-minute time period represents a break-point, and the concentrations slowly decrease after this point. 
Table 1. Patients’ age, weight and evaluations of anxiety and pain using visual analogue scales.

\begin{tabular}{cccc}
\hline & Propofol & Propofol and alfentanil & p value \\
\hline Age (years) & $57(14)$ & $56(13)$ & 0.68 \\
Weight $(\mathrm{kg})$ & $70(14)$ & $72(14)$ & 0.29 \\
Preoperative anxiety & $25(15-45 ; 0-95)$ & $28(10-47 ; 0-85)$ & 0.92 \\
Peroperative anxiety & $7(3-13 ; 0-60)$ & $5(3-13 ; 0-75)$ & 0.55 \\
Peroperative pain & $7(2-15 ; 0-83)$ & $5(2-18 ; 0-65)$ & 0.42 \\
\hline
\end{tabular}

Age and weight: values are mean (SD). Anxiety and pain: values are median (IQR and range). Intensity of anxiety and pain is rated according to the most anxiety and pain experienced during the procedure $(0=$ no anxiety/pain; $100=$ intolerable anxiety/pain $)$.

Table 2. Multiple regression model showing the association between details from the procedures and the patients and the mean estimated concentrations of propofol at the effect site (mean concentration for the first 20 minutes of procedure).

\begin{tabular}{ccccc}
\hline & & Propofol & \multicolumn{2}{c}{ Propofol and alfentanil } \\
\hline Variables & B-coef & p value & B-coef & p value \\
\hline Type of operation & & & & 0.11 \\
Incontinence & 0.20 & $\leq 0.01$ & 0.15 & 0.47 \\
Anterior repair & 0.20 & $<0.01$ & -0.15 & 0.67 \\
Posterior repair & $<0.01$ & 0.75 & 0.15 & 0.17 \\
Combinations & $<0.01$ & 0.52 & 0.23 & $\leq 0.01$ \\
Alfentanil (yes/no) & & & -0.40 & 0.14 \\
Preoperative anxiety & $<-0.01$ & 0.85 & $<0.01$ & 0.72 \\
Peroperative anxiety & $<0.01$ & 0.75 & $<0.01$ & 0.76 \\
Peroperative peak pain & $<0.01$ & 0.32 & $<-0.01$ & 0.19 \\
Age (years) & $<-0.01$ & 0.38 & -0.01 & $\leq 0.01$ \\
Weight (kg) & 0.01 & $<0.01$ & 0.01 & 0.23 \\
ASA II + III & $<-0.01$ & 0.23 & -0.16 & \\
\hline
\end{tabular}

B-coef $=$ coefficients of the independent variables. ASA = American Society of Anesthesiologists. For concentrations of propofol ( $\mu$ g/ml), model overall $r=0.69$; adj $r^{2}=0.48$. For concentrations of propofol and alfentanil, model overall $r=0.70$; adj $r^{2}=0.43$. Significant independent variables for the overall model are underlined. Non-significant variables are displayed with coefficients and p-values representing the effect they had when they were excluded from the model. A stepwise backwards approach was used.

The ten highest peak concentrations of alfentanil were not associated with respiratory events. The $\mathrm{C}_{\mathrm{e}}$ for those ten patients were significantly higher than for the ten who developed respiratory events $(0.12$ (0.02) compared with $0.08(0.01) \mu \mathrm{g} / \mathrm{ml} ; \mathrm{p}<0.01)$. The $\mathrm{C}_{\mathrm{e}}$ for alfentanil in the ten patients who used the pump most often (dose count) were higher than those for the ten with respiratory impairments $(0.11(0.09) \mu \mathrm{g} / \mathrm{ml}$; p $<0.01)$. Figure 2 shows the $\mathrm{C}_{\mathrm{e}}$ for alfentanil from all patients in the propofol/alfentanil group and from the patients with respiratory depression. However, comparison of the $\mathrm{C}_{\mathrm{e}}$ of the two drugs (Figure 1 and Figure 2) indicates that the mean peak concentration of propofol in patients who developed adverse effects was higher, although not significantly so. The combination of propofol and alfentanil, $C_{e}>2.0$ and $>0.05 \mu \mathrm{g} / \mathrm{ml}$, respectively, seemed to increase the risk of respiratory events. Logistic regression also showed that the combination of $C_{e}$ for propofol and alfentanil contributed to the respiratory events in the ten patients, along with age. The model explains the impaired respiratory functions using the variables alfentanil $\mathrm{C}_{e}(\mathrm{p}=0.02)$, propofol $\mathrm{C}_{\mathrm{e}}(\mathrm{p}=0.04)$, and patients' age $(p=0.03)$. Coefficients for alfentanil $C_{e}$, propofol $C_{e}$ and patients' age were: 54.8; 0.191; 0.012, respectively. 


\section{Discussion}

The new findings in this study are that we have isolated some of the factors that contribute to patients' dosing patterns from PCS systems. We can also explain the risk of respiratory impairment, as the use of alfentanil in itself is a risk factor, rather than the concentrations of drugs at the effect site. Anterior repair and treatment for incontinence are known to be unpleasant, and it is sometimes hard to get adequate local anaesthesia for these operations. The relationship between them and increased concentrations of propofol seems logical. The influence of weight on $C_{e}$ may be explained by the fact that increased body weight led to a more intense use of the PCS pump during induction, or from the fact that nine of the patients in the study had weights between 102 and $121 \mathrm{~kg}$, and the impact from these patients on the regression analysis could be enough to explain the impact of weight on propofol $\mathrm{C}_{\mathrm{e}}$.

The multiple regression analysis indicated that operative pain and anxiety had no impact on the concentrations of drugs. This was surprising, as our clinical experience has shown that patients who are anxious preoperatively are given, and usually ask for, larger doses of sedatives if they are about to undergo an unpleasant procedure. When target-controlled infusion was used for sedation, results similar to sedation for oocyte retrieval [14] were reported, as well as the opposite for colonoscopy [15]. A major distinction between these and the present data could explain the main difference between PCS and anaesthetist-controlled sedation: the doses required for a target-controlled infusion are not correlated with a patient's need to be sedated, but are related to a measured degree of sedation.

The main reason for the choice of PCS is that the model includes built-in feedback, and patients adjust the doses according to their need. Self-dosing with sedatives tends to result in fewer doses of drugs being used than when anaesthetists give sedatives [16] [17]. However, overdosing can still occur with PCS. When the patient stops pressing the button, the concentration continues to increase for a period of time: if a patient presses the button for 2 minutes (11 doses), the concentration will increase by about $20 \%$ for the next 1.5 minutes. After the patient has stopped requesting more, not only will the concentration of propofol increase, but also that of alfentanil. Smaller doses of alfentanil could have been considered in our study, as the accumulated doses then would have had less impact on respiratory function. Remifentanil could also have been considered as an adjunct to propofol because it has a kinetic profile similar to that of propofol, and the risk of late respiratory impairment is low. The main reason that remifentanil was not used in the present study is that the procedures were done in an out-patient clinic, and remifentanil was not considered suitable for use outside the operating theatre. Alfentanil has also been recommended for use with propofol as PCS instead of remifentanil [9], and has been reported to serve well for the treatment of renal stones in the hands of non-anaesthetists [18].

Pharmacokinetic simulation has rarely, if ever, been used for PCS studies. The present simulation clearly shows that a mixture of two drugs with different pharmacokinetic profiles in the same syringe might induce serious respiratory effects. The highest concentration of propofol was detected after only five minutes (Figure 1). The respiratory depressant action of alfentanil that was added at this time, together with the still-increasing concentration of alfentanil, caused some cases of respiratory depression and even apnoea. At this stage, the local anaesthetic had been infiltrated by the surgeon, and the procedure started almost without stimuli. The concentration of alfentanil remained high for 30 minutes, but the risk of later respiratory impairment was probably reduced by the decreasing concentration of propofol as the patients' requirements for new doses decreased as the procedure progressed under effective local anaesthesia. Surveys of PCS that have used the combination of propofol and an opioid have also reported events that might compromise safety [8] [9].

The logistic regression model showed that age and $\mathrm{C}_{\mathrm{e}}$ for both propofol and alfentanil were related to the respiratory impairment in the ten affected patients. There are differences between simulated and measured concentrations, and different time frames for target concentrations [19] [20], and the individual clinical response to a specific $\mathrm{C}_{\mathrm{e}}$ is hard to predict. Inclusion of an operator control for loss of consciousness may reduce the impact of biological variability if a target-controlled infusion (TCI) of propofol is used [21], but its use with TCI or PCS in clinical practise is unclear. The evidence of age as an explanatory variable is confirmed, as the use of TCI anaesthesia has shown that age affects the association between the $C_{e}$ of propofol and the loss of, and return to, consciousness [22]. PCS with propofol could be improved by giving opioids manually (and decrease the dose of opioids at a certain age) or by adding pharmacokinetic calculation software to a PCS device. Further studies are required.

The present study has limitations. First, our regression model points out some of the variables that influence 
patients dosing themselves, but the $\mathrm{r}^{2}$ of 0.48 indicates that other variables contribute largely to this. Evaluation of the VAS for anxiety and pain is common, but may not include all the aspects of anxiety measured by specific measurements, such as the Beck Anxiety Inventory scores [15], or Spielberger's State-Trait Anxiety Inventory [23]. Coping strategies may also influence patients' behaviour and the number of requests for sedatives. We have not evaluated this aspect.

Second, 10/155 patients in the logistic regression analysis of the respiratory effects of PCS is a small number, which reduces our predictive possibilities. The role of alfentanil is an interesting issue, as propofol used alone caused no respiratory events that required intervention. Factors other than drugs and age are likely to be confounding interactively. Enrolment of a larger study group would enable an analysis of greater complexity and sensitivity. However, to our knowledge, similar evaluations are rare.

Third, to adjust for unknown independent variables, a more homogeneous study group would make the results more accurate. Further and repeated surveys are needed for the refinement of the PCS system. Increased suitability could thereby be achieved through adjusted bolus doses or maximum dose counts/minutes according to age, weight, or other characteristics. Dose-adjusted PCS systems have not been evaluated.

\section{Conclusion}

In conclusion, the patients' weight and the type of surgery performed were associated with the $\mathrm{C}_{\mathrm{e}}$ of propofol; this knowledge could be used for the refinement of the doses given through PCS. Because the pharmacokinetic profiles of propofol and alfentanil differed, the alfentanil effect became predominant during the time course of sedation. In order to reduce the risk of early and late respiratory depression, for operations in which local anaesthetics are used, we propose that alfentanil should not be added to propofol in the same syringe.

\section{Support and Presentation}

This work was financially supported by the Department of Anaesthesiology and Intensive Care, Linköping University Hospital, 58185 Linköping, Sweden.

Preliminary data were presented as a poster at the European Society of Anaesthesiology (ESA) Euroanaesthesia, 1 June 2014, Stockholm.

\section{References}

[1] Pollock, H., Forman, S., Pollock, T. and Raccasi, M. (2013) Conscious Sedation/Local Anesthesia in the Office-Based Surgical and Procedural Facility. Clinics in Plastic Surgery, 40, 383-388. http://dx.doi.org/10.1016/j.cps.2013.04.014

[2] (2002) Practice Guidelines for Sedation and Analgesia by Non-Anesthesiologists. Anesthesiology, 96, 1004-1017. http://dx.doi.org/10.1097/00000542-200204000-00031

[3] Knape, J.T., Adriaensen, H., van Aken, H., Blunnie, W.P., Carlsson, C., Dupont, M. and Pasch, T., Board of Anaesthesiology of the European Union of Medical Specialists (2007) Guidelines for Sedation and/or Analgesia by NonAnaesthesiology Doctors. European Journal of Anaesthesiology, 24, 563-567. http://dx.doi.org/10.1017/S0265021506002092

[4] Wehrmann, T. and Riphaus, A. (2008) Sedation with Propofol for Interventional Endoscopic Procedures: A Risk Factor Analysis. Scandinavian Journal of Gastroenterology, 43, 368-374. http://dx.doi.org/10.1080/00365520701679181

[5] Agostoni, M., Fanti, L., Arcidiacono, P.G., Gemma, M., Strini, G., Torri, G. and Testoni, P.A. (2007) Midazolam and Pethidine versus Propofol and Fentanyl Patient Controlled Sedation/Analgesia for Upper Gastrointestinal Tract Ultrasound Endoscopy: A Prospective Randomized Controlled Trial. Digestive and Liver Disease, 39, 1024-1029. http://dx.doi.org/10.1016/j.dld.2007.08.004

[6] Hwang, J., Jeon, Y., Park, H.P., Lim, Y.J. and Oh, Y.S. (2005) Comparison of Alfetanil and Ketamine in Combination with Propofol for Patient-Controlled Sedation during Fiberoptic Bronchoscopy. Acta Anaesthesiologica Scandinavica, 49, 1334-1338. http://dx.doi.org/10.1111/j.1399-6576.2005.00842.x

[7] Mandel, J.E., Tanner, J.W., Lichtenstein, G.R., Metz, D.C., Katzka, D.A., Ginsberg, G.G. and Kochman, M.L. (2008) A Randomized, Controlled, Double-Blind Trial of Patient-Controlled Sedation with Propofol/Remifentanil versus Midazolam/Fentanyl for Colonoscopy. Anesthesia and Analgesia, 106, 434-439. http://dx.doi.org/10.1213/01.ane.0000297300.33441.32

[8] Joo, H.S., Perks, W.J., Kataoka, M.T., Errett, L., Pace, K. and Honey, R.J. (2001) A Comparison of Patient-Controlled Sedation Using Either Remifentanil or Remifentanil-Propofol for Shock Wave Lithotripsy. Anesthesia Analgesia, 93, 1227-1232. http://dx.doi.org/10.1097/00000539-200111000-00037 
[9] Mazanikov, M., Udd, M., Kylanpaa, L., Mustonen, H., Lindstrom, O., Halttunen, J., Farkkila, M. and Poyhia, R. (2012) Patient-Controlled Sedation for ERCP: A Randomized Double-Blind Comparison of Alfentanil and Remifentanil. Endoscopy, 44, 487-492. http://dx.doi.org/10.1055/s-0031-1291655

[10] Nilsson, A., Nilsson, L., Ustaal, E. and Sjoberg, F. (2012) Alfentanil and Patient-Controlled Propofol Sedation—Facilitate Gynaecological Outpatient Surgery with Increased Risk of Respiratory Events. Acta Anaesthesiologica Scandinavica, 56, 1123-1129. http://dx.doi.org/10.1111/j.1399-6576.2012.02749.x

[11] Schnider, T.W., Minto, C.F., Gambus, P.L., Andresen, C., Goodale, D.B., Shafer, S.L. and Youngs, E.J. (1998) The Influence of Method of Administration and Covariates on the Pharmacokinetics of Propofol in Adult Volunteers. Anesthesiology, 88, 1170-1182. http://dx.doi.org/10.1097/00000542-199805000-00006

[12] Schnider, T.W., Minto, C.F., Shafer, S.L., Gambus, P.L., Andresen, C., Goodale, D.B. and Youngs, E.J. (1999) The Influence of Age on Propofol Pharmacodynamics. Anesthesiology, 90, 1502-1516. http://dx.doi.org/10.1097/00000542-199906000-00003

[13] Scott, J.C. and Stanski, D.R. (1987) Decreased Fentanyl and Alfentanil Dose Requirements with Age. A Simultaneous Pharmacokinetic and Pharmacodynamic Evaluation. The Journal of Pharmacology and Experimental Therapeutics, 240, 159-166. http://dx.doi.org/10.1097/00132586-198710000-00044

[14] Hong, J.Y., Jee, Y.S. and Luthardt, F.W. (2005) Comparison of Conscious Sedation for Oocyte Retrieval between Low-Anxiety and High-Anxiety Patients. Journal of Clinical Anesthesia, 17, 549-553. http://dx.doi.org/10.1016/j.jclinane.2005.01.008

[15] Chung, K.C., Juang, S.E., Lee, K.C., Hu, W.H., Lu, C.C., Lu, H.F. and Hung, K.C. (2013) The Effect of Pre-Procedure Anxiety on Sedative Requirements for Sedation during Colonoscopy. Anaesthesia, 68, 253-259. http://dx.doi.org/10.1111/anae.12087

[16] Nilsson, A., Steinvall, I., Bak, Z. and Sjoberg, F. (2008) Patient Controlled Sedation Using a Standard Protocol for Dressing Changes in Burns: Patients' Preference, Procedural Details and a Preliminary Safety Evaluation. Burns, 34, 929-934. http://dx.doi.org/10.1016/j.burns.2008.04.002

[17] Crepeau, T., Poincloux, L., Bonny, C., Lighetto, S., Jaffeux, P., Artigue, F., Walleckx, P., Bazin, J.E., Dapoigny, M. and Bommelaer, G. (2005) Significance of Patient-Controlled Sedation during Colonoscopy. Results from a Prospective Randomized Controlled Study. Gastroentérologie Clinique et Biologique, 29, 1090-1096. http://dx.doi.org/10.1016/S0399-8320(05)82172-4

[18] Ortiz, A.C., Atallah, A.N., Matos, D. and da Silva, E.M.K. (2014) Intravenous versus Inhalational Anaesthesia for Paediatric Outpatient Surgery. The Cochrane Database of Systematic Reviews, 2, Article ID: CD009015. http://dx.doi.org/10.1002/14651858.cd009015.pub2

[19] Frolich, M.A., Dennis, D.M., Shuster, J.A. and Melker, R.J. (2005) Precision and Bias of Target Controlled Propofol Infusion for Sedation. British Journal of Anaesthesia, 94, 434-437. http://dx.doi.org/10.1093/bja/aei081

[20] Enlund, M. (2008) TCI: Target Controlled Infusion, or Totally Confused Infusion? Call for an Optimised Population Based Pharmacokinetic Model for Propofol. Upsala Journal of Medical Sciences, 113, 161-170. http://dx.doi.org/10.3109/2000-1967-222

[21] Mandel, J.E. and Sarraf, E. (2012) The Variability of Response to Propofol Is Reduced When a Clinical Observation Is Incorporated in the Control: A Simulation Study. Anesthesia and Analgesia, 114, 1221-1229. http://dx.doi.org/10.1213/ANE.0b013e31824cb853

[22] Koo, B.N., Lee, J.R., Noh, G.J., Lee, J.H., Kang, Y.R. and Han, D.W. (2012) A Pharmacodynamic Analysis of Factors Affecting Recovery from Anesthesia with Propofol-Remifentanil Target Controlled Infusion. Acta pharmacologica Sinica, 33, 1080-1084. http://dx.doi.org/10.1038/aps.2012.85

[23] Kil, H.K., Kim, W.O., Chung, W.Y., Kim, G.H., Seo, H. and Hong, J.Y. (2012) Preoperative Anxiety and Pain Sensitivity Are Independent Predictors of Propofol and Sevoflurane Requirements in General Anaesthesia. British Journal of Anaesthesia, 108, 119-125. http://dx.doi.org/10.1093/bja/aer305 